
\title{
25 Research Soure \\ Xylitol Enhances Synthesis of Propionate in Colon via Cross-feeding of Gut Microbiota
}

\author{
Shasha Xiang \\ Kun Ye \\ Mian Li \\ Jian Ying \\ Huanhuan Wang \\ Jianzhong Han \\ Lihua Shi \\ Jie Xiao \\ Yubiao Shen \\ Xiao Feng \\ Xuan Bao \\ Yiqing Zheng \\ Yin Ge \\ Yalin Zhang \\ Chang Liu \\ Jie Chen \\ Yuewen Chen \\ Shiyi Tian \\ Xuan Zhu
}

\section{Video Byte}

Keywords: intestinal microorganisms, xylitol, in vitro colonic simulation system, CDMN, cross-feeding, sweetener, probiotic, gut microbiome, Microbiome

Posted Date: October 15th, 2021

DOI: https://doi.org/10.21203/rs.3.rs-969885/v1

License: (c) (i) This work is licensed under a Creative Commons Attribution 4.0 International License. Read Full License 


\section{Abstract}

Xylitol is a popular sugar substitute that exists in small amounts in fruits, vegetables, and brans. In addition to its use as a sweetener, xylitol has also gained notoriety for its prebiotic-like activity. Xylitol is digestible by gut microbes and promotes the proliferation of beneficial bacteria and the production of short-chain fatty acids (SCFAs), which are essential for gut health. To better understand the mechanisms behind these effects, researchers studied the effects of xylitol both in mice and in an in vitro colon simulation system (CDMN). Sequencing revealed that in vivo and in vitro, xylitol did not significantly influence the structure of the gut microbiome. It did, however, increase the amount of SCFAs in the gut mucosa. Cross-feeding - a relationship in which one organism consumes metabolites excreted by another - was observed among bacterial species for xylitol, and key enzymes in xylitol metabolism were found in two bacterial species, Bacteroides and Lachnospiraceae. Xylitol also increased the production of propionate, a metabolite that can lower gut $\mathrm{pH}$ and restrict the growth of some bacteria. While further study in humans is needed, these results suggest that xylitol may promote gut health through its ability to boost the production of beneficial enzymes and SCFAs. 